\title{
The Higgs singlet extension at LHC Run 2
}

\section{G. Chalons}

Laboratoire de Physique Subatomique et de Cosmologie

Université Grenoble-Alpes, CNRS/IN2P3

53 Rue des Martyrs

F-38026 Grenoble Cedex, France

E-mail: chalons@lpsc.in2p3.fr

\section{López-Val}

Center for Cosmology, Particle Physics and Phenomenology CP3

Université catholique de Louvain

Chemin du Cyclotron 2, B-1348 Louvain-la-Neuve, Belgium

E-mail: david.lopezval@uclouvain.be

\section{T. Robens*}

IKTP, Technische Universität Dresden

Zellescher Weg 19, D-01069 Dresden, Germany

E-mail: Tania.Robens@tu-dresden.de

\section{T. Stefaniak}

Department of Physics and Santa Cruz Institute for Particle Physics University of California, Santa Cruz, CA 95064, USA

E-mail: tistefan@ucsc.edu

We discuss the current status of theoretical and experimental constraints on the real Higgs singlet extension of the Standard Model. For the second neutral (non-standard) Higgs boson the mass range up to $1 \mathrm{TeV}$ accessible at past and current collider experiments is considered. We furthermore discuss electroweak corrections to the $H \rightarrow h h$ partial decay width within this model.

CP3-16-34, LPSC16110

XXIV International Workshop on Deep-Inelastic Scattering and Related Subjects

11-15 April, 2016

DESY Hamburg, Germany

\footnotetext{
*Speaker.
} 


\section{The model}

In this work we consider the simplest extension of the Standard Model (SM) Higgs sector, where an additional real scalar field is added $[1,2,3]$. The model contains a complex $S U(2)_{L}$ doublet, in the following denoted by $\Phi$, and a real scalar $S$ which is a singlet under the SM gauge group. The most general renormalizable Lagrangian compatible with an additional $Z_{2}$ symmetry is then given by $\mathscr{L}_{s}=\left(D^{\mu} \Phi\right)^{\dagger} D_{\mu} \Phi+\partial^{\mu} S \partial_{\mu} S-V(\Phi, S)$, with the scalar potential

$$
V(\Phi, S)=-m^{2} \Phi^{\dagger} \Phi-\mu^{2} S^{2}+\lambda_{1}\left(\Phi^{\dagger} \Phi\right)^{2}+\lambda_{2} S^{4}+\lambda_{3} \Phi^{\dagger} \Phi S^{2} .
$$

In the unitary gauge, the Higgs fields are given by $\Phi \equiv\left(0 \frac{\tilde{h}+v}{\sqrt{2}}\right)^{T}, S \equiv \frac{h^{\prime}+v_{s}}{\sqrt{2}}$, with $v, v_{s}$ denoting the non-zero vacuum expectation values of the doublet and singlet. Physically, the above potential leads to a mixing between the gauge eigenstates, related via the mixing angle $\alpha$ according to $h=$ $c_{\alpha} \tilde{h}-s_{\alpha} h^{\prime}, H=s_{\alpha} \tilde{h}+c_{\alpha} h^{\prime}$, where we used the shorthand notation $s_{\alpha}\left(c_{\alpha}\right) \equiv \sin \alpha(\cos \alpha)$. We here use the convention that $m_{h} \leq m_{H}$, and choose as input parameters $m_{h}, m_{H}, \sin \alpha, v, \tan \beta \equiv \frac{v}{v_{s}}$, where $v \sim 246 \mathrm{GeV}$. In addition, one of the scalar masses is fixed to $\sim 125 \mathrm{GeV}$, where we distinguish between the high-mass $\left(m_{h} \sim m_{h, \mathrm{SM}}\right)$ and low-mass $\left(m_{H} \sim m_{h, \mathrm{SM}}\right)$ scenario. The above mixing also leads to the familiar rescaling of the SM-like Higgs couplings at tree level by $\sin \alpha(\cos \alpha)$ for $\mathrm{h}(\mathrm{H})$, with respect to the couplings for a SM Higgs boson of that mass.

\section{Parameter constraints and predictions at the LHC Run 2}

We refer the reader to $[4,5,6]$ for a detailed discussion of the individual constraints. Vacuum stability, perturbative unitarity, perturbativity of the couplings, agreement with electroweak precision observables have been explicitly discussed in the above references; constraints from the $W$-boson mass measurement follow [7]. In [6], previous results were updated especially with regard to the latest LHC limits and Higgs signal strength measurements [8], using the public tools HiggsBounds (version 4.3.1) [9, 10,11] and HiggsSignals (version 1.4.0) [12]. A summary of all constraints on the maximal mixing angle $\sin \alpha$ is shown in Fig. 1. Production cross-sections for the $14 \mathrm{TeV}$ LHC, after all constraints have been taken into account, are shown in Fig. 2 for the high-mass range. Specific benchmarks for all mass ranges have been presented in $[6]^{1}$.

\section{Renormalization}

The complete electroweak renormalization of the singlet model has been presented in [14], and we refer the reader to this reference for explicit details. Here we only want to point to two major features of our scheme setup.

Non-linear gauge fixing We use a non-linear gauge fixing, specified by

$$
\mathscr{L}_{G F}=-\frac{1}{\xi_{W}} F^{+} F^{-}-\frac{1}{2 \xi_{Z}}\left|F^{Z}\right|^{2}-\frac{1}{2 \xi_{A}}\left|F^{A}\right|^{2},
$$

\footnotetext{
${ }^{1}$ See also [13].
} 


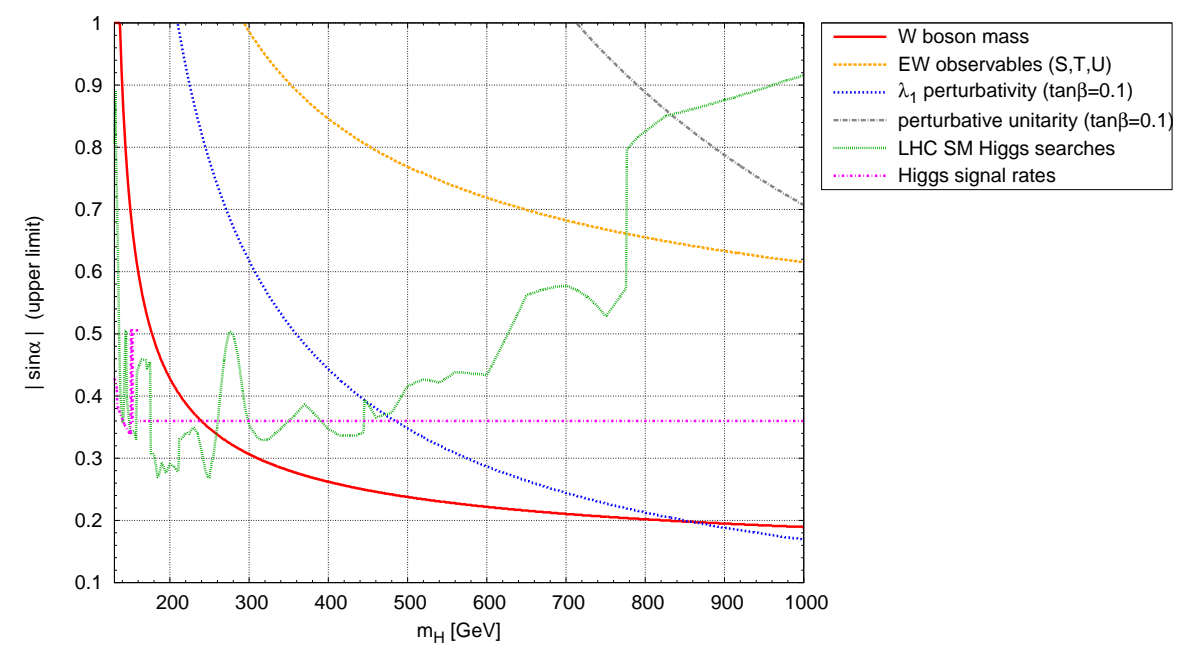

Figure 1: Maximal allowed values for $|\sin \alpha|$ in the high mass region, $m_{H} \in[130,1000] \mathrm{GeV}$, from NLO calculations of the $W$ boson mass (red, solid) [7], electroweak precision observables (EWPOs) tested via the oblique parameters $S, T$ and $U$ (orange, dashed), perturbativity of the RG-evolved coupling $\lambda_{1}$ (blue, dotted), evaluated for an exemplary choice $\tan \beta=0.1$, perturbative unitarity (grey, dash-dotted), direct LHC Higgs searches (green, dashed), and the Higgs signal strength (magenta, dash-dotted). Taken from [6].

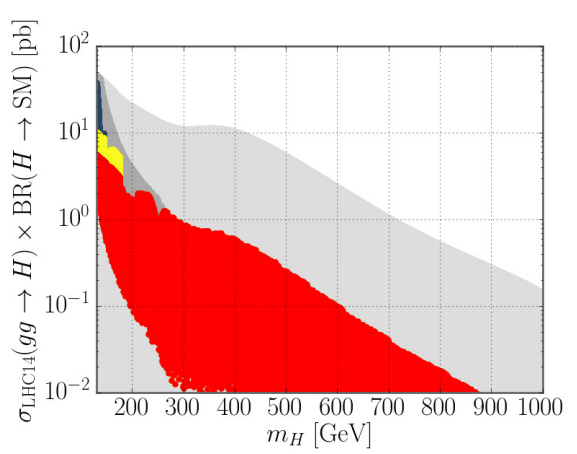

(a) Heavy Higgs signal rate with SM particles in the final state for the $\mathrm{LHC}$ at $14 \mathrm{TeV}$.

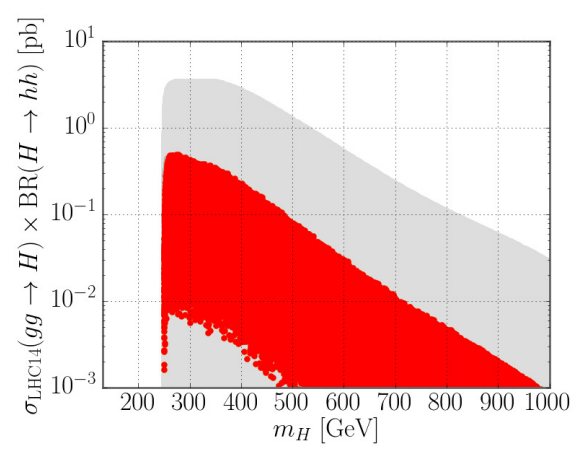

(b) Heavy Higgs signal rate with light Higgs bosons in the final state for the $\mathrm{LHC}$ at $14 \mathrm{TeV}$.

Figure 2: Production cross-sections at a $14 \mathrm{TeV}$ LHC, for a heavy Higgs $H$ decaying into SM particles (left) or $h h$ final states (right); for the latter, electroweak corrections have not been included. Cross sections stem from a simple rescaling of production cross sections presented in [13]. Red and yellow regions correspond to agreement with the Higgs signal strength measurements at the $1 \sigma$ and $2 \sigma$ level, respectively, blue points comply with direct experimental searches but do not agree with the Higgs signal strength within $2 \sigma$. Taken from [6]. 
where the functions $F$ depend non-linearly on the Higgs and gauge fields and are given by Eqns. (21)-(23) of [14]. The gauge-fixing terms explicitly depend on the non-linear gauge-fixing quantities $\tilde{\delta}_{i}$. We perform our implementation of the singlet model using SLOops (see e.g. $[15,16]$ ).

Gauge-parameter independent physical results We have studied different schemes and explicitly tested gauge-fixing parameter dependence. An improved On-shell prescription leads to gaugeparameter independent predictions for the one-loop corrections to $\Gamma_{H \rightarrow h h}$ :

$$
\delta m_{h H}^{2}=\left.\operatorname{Re} \Sigma_{h H}\left(p_{*}^{2}\right)\right|_{\xi_{W}=\xi_{Z}=1, \tilde{\delta}_{i}=0} \quad \text { with } \quad p_{*}^{2}=\frac{m_{h}^{2}+m_{H}^{2}}{2},
$$

This prescription coincides with the discussion in [16] in the context of supersymmetry, and can also be related to the so-called pinch technique (see e.g. [17]).

We rely on two independent implementations of the model ${ }^{2}$. Once all present constraints on the model are included, we find mild NLO corrections, typically of few percent, and with theoretical uncertainties on the per mille level. Sample results for the one-loop eletroweak corrections to the decay width $\Gamma_{H \rightarrow h h}$ are displayed in Fig. 3.

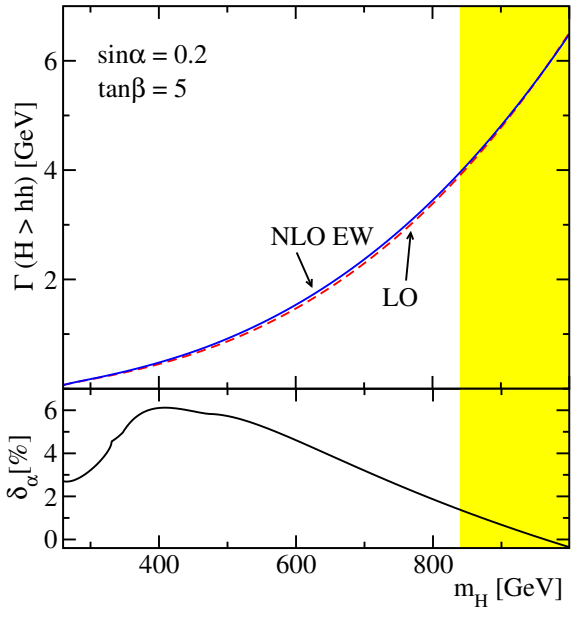

(a) $m_{h}=125.09 \mathrm{GeV}$

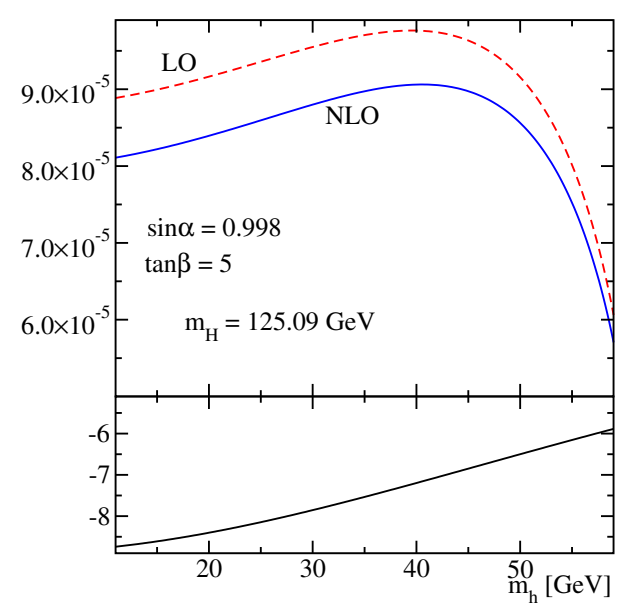

(b) $m_{H}=125.09 \mathrm{GeV}$

Figure 3: NLO corrections to the $H \rightarrow h h$ partial decay width, for fixed $\sin \alpha, \tan \beta$ values and $m_{h}$ (left) or $m_{H}$ (right) being the $125 \mathrm{GeV}$ resonance measured at the LHC, as a function of the second scalar mass. We display the total decay width for $H \rightarrow h h$, as well as the relative correction in the $\alpha_{\mathrm{em}}$ input scheme for the electroweak parameters (see [14] for details). The yellow region is excluded by perturbativity of the couplings. Note: $\tan \beta$ is defined as $\frac{v_{s}}{v}$ in this case, in contrast to the definitions given above. Taken from [14].

\section{Acknowledgements}

TR and TS want to thank M. Gouzevitch and M. Slawinska for a useful discussion regarding one of the figures presented in [6]. ${ }^{3}$

\footnotetext{
${ }^{2}$ See [14] for a complete description of the computational setup.

${ }^{3}$ Funding is acknowledged from the F.R.S.-FNRS "Fonds de la Recherche Scientifique", the Theory- LHC-France initiative of CNRS/IN2P3, the U.S. Department of Energy grant number DE-SC0010107 and the Alexander von Humboldt foundation.
} 


\section{References}

[1] R. Schabinger and J. D. Wells, A Minimal spontaneously broken hidden sector and its impact on Higgs boson physics at the large hadron collider, Phys.Rev. D72 (2005) 093007, [hep-ph / 0509209 ].

[2] B. Patt and F. Wilczek, Higgs-field portal into hidden sectors, hep-ph/0605188.

[3] M. Bowen, Y. Cui and J. D. Wells, Narrow trans-TeV Higgs bosons and $H \rightarrow$ hh decays: Two LHC search paths for a hidden sector Higgs boson, JHEP 0703 (2007) 036, [hep-ph / 0701035 ].

[4] G. M. Pruna and T. Robens, The Higgs Singlet extension parameter space in the light of the LHC discovery, Phys.Rev. D88 (2013) 115012, [1303.1150].

[5] T. Robens and T. Stefaniak, Status of the Higgs Singlet Extension of the Standard Model after LHC Run 1, Eur. Phys. J. C75 (2015) 104, [1501.02234].

[6] T. Robens and T. Stefaniak, LHC Benchmark Scenarios for the Real Higgs Singlet Extension of the Standard Model, Eur. Phys. J. C76 (2016) 268, [1601.07880].

[7] D. Lopez-Val and T. Robens, Delta $r$ and the W-boson mass in the Singlet Extension of the Standard Model, Phys.Rev. D90 (2014) 114018, [1406.1043].

[8] ATLAS and CMS Collaborations, Measurements of the Higgs boson production and decay rates and constraints on its couplings from a combined ATLAS and CMS analysis of the LHC pp collision data at $\sqrt{s}=7$ and $8 \mathrm{TeV}$.

[9] P. Bechtle, O. Brein, S. Heinemeyer, G. Weiglein and K. E. Williams, HiggsBounds: Confronting Arbitrary Higgs Sectors with Exclusion Bounds from LEP and the Tevatron, Comput. Phys. Commun. 181 (2010) 138, [0811.4169].

[10] P. Bechtle, O. Brein, S. Heinemeyer, G. Weiglein and K. E. Williams, HiggsBounds 2.0.0: Confronting Neutral and Charged Higgs Sector Predictions with Exclusion Bounds from LEP and the Tevatron, Comput. Phys. Commun. 182 (2011) 2605, [1102 . 1898 ].

[11] P. Bechtle, O. Brein, S. Heinemeyer, O. Stål, T. Stefaniak et al., HiggsBounds-4: Improved Tests of Extended Higgs Sectors against Exclusion Bounds from LEP, the Tevatron and the LHC, Eur. Phys. J. C 74 (2013) 2693, [1311. 0055$].$

[12] P. Bechtle, S. Heinemeyer, O. Stål, T. Stefaniak and G. Weiglein, HiggsSignals: Confronting arbitrary Higgs sectors with measurements at the Tevatron and the LHC, Eur.Phys.J. C74 (2014) 2711, [1305.1933].

[13] The LHC Higgs Cross Section Working Group, CERN Yellow Report 4, .

[14] F. Bojarski, G. Chalons, D. Lopez-Val and T. Robens, Heavy to light Higgs boson decays at NLO in the Singlet Extension of the Standard Model, JHEP 02 (2016) 147, [1511.08120].

[15] F. Boudjema, A. Semenov and D. Temes, Self-annihilation of the neutralino dark matter into two photons or a Z and a photon in the MSSM, Phys. Rev. D72 (2005) 055024, [hep-ph/ 0507127 ].

[16] N. Baro and F. Boudjema, Automatised full one-loop renormalisation of the MSSM II: The chargino-neutralino sector, the sfermion sector and some applications, Phys. Rev. D80 (2009) 076010, [0906.1665].

[17] J. R. Espinosa and Y. Yamada, Scale independent and gauge independent mixing angles for scalar particles, Phys. Rev. D67 (2003) 036003, [hep-ph/ 0207351$].$ 